CTP-2509

\title{
Simulations of Discrete Quantum Systems in Continuous Euclidean Time
}

\author{
B.B. Beard and U.-J. Wiese \\ Center for Theoretical Physics, \\ Laboratory for Nuclear Science, and Department of Physics \\ Massachusetts Institute of Technology \\ Cambridge, Massachusetts 02139, U.S.A.
}

(October 25, 2018)

\begin{abstract}
Path integrals are usually formulated in discrete Euclidean time using the Trotter formula. We propose a new method to study discrete quantum systems, in which we work directly in the Euclidean time continuum. The method is of general interest and can be applied to studies of quantum spin systems, lattice fermions, and in principle also lattice gauge theories. Here it is applied to the Heisenberg quantum antiferromagnet using a continuous-time version of a loop cluster algorithm. The computational advantage of this algorithm is exploited to confirm the predictions of chiral perturbation theory in the extreme low temperature regime, down to $T=0.01 \mathrm{~J}$. A fit of the low-energy parameters of chiral perturbation theory gives excellent agreement with previous results and with experiments.
\end{abstract}

75.30.D,75.10.J,02.70.Lq,31.15.K,71.10.F

Typeset using REVTEX 
The path integral for a general quantum system is usually formulated in discrete Euclidean time. Farhi and Gutmann pointed out that this is not necessary, since the path integral is well-defined in continuous time [1]. For example, for a non-relativistic particle in coordinate space, each trajectory in the path integral resembles that of a particle undergoing Brownian motion. Due to the continuum of position eigenstates, defining the corresponding Wiener measure seems to require discrete time. However, one can always work in a countable basis of the Hilbert space: one can put the particle in a large box and work in the discrete basis of momentum eigenstates, for example. The important point is that then the trajectories in the path integral do not resemble Brownian motion. Instead they consist of segments for which the system is in a basis state for a finite time, with sporadic discrete transitions between basis states. Consequently, for discrete quantum systems the path integral can be formulated directly in continuous time. This insight can be applied, for example, to quantum spin systems, lattice fermions, and lattice gauge theories with a compact gauge group.

In the continuous-time formulation a path is characterized by transition times and information about which states are connected at the transitions. This picture allows the path integral to be sampled numerically without having to store information about the individual time slices whose number diverges in the time-continuum limit. In the discrete-time approximation one must always exercise care in extrapolating the results to the time-continuum limit. The approach advocated here cuts this Gordian knot by operating directly in this limit. This completely eliminates the most severe systematic error in these calculations.

As an example we consider quantum spin systems. Conventional approaches to handling these systems rely on a discrete-time formalism. Consider, as a concrete example, the Hamiltonian for the Heisenberg quantum antiferromagnet

$$
H=J \sum_{x, \mu} \vec{S}_{x} \cdot \vec{S}_{x+\hat{\mu}}
$$

where $\vec{S}_{x}=\frac{1}{2} \vec{\sigma}_{x}$ is a spin $1 / 2$ operator associated with the site $x$ of a $d$-dimensional hypercubic lattice with spacing $a$. The interaction is between nearest neighbors; $\hat{\mu}$ is the unit 
vector in the $\mu$-direction. For an antiferromagnet the coupling constant $J$ is positive. Since this Hamiltonian comprises non-commuting terms, the explicit evaluation of the associated partition function is problematical. Suzuki is credited with showing how the Trotter formula can be applied to segregate these non-commuting terms into separate time slices, with the approximation becoming exact in the continuum limit [2]. For example, the Hamiltonian of a $1 \mathrm{D}$ quantum spin chain can be decomposed into two terms, $H=H_{1}+H_{2}$, each of which comprises only commuting terms

$$
H_{1}=J \sum_{x=2 m} \vec{S}_{x} \cdot \vec{S}_{x+\hat{1}}, \quad H_{2}=J \sum_{x=2 m+1} \vec{S}_{x} \cdot \vec{S}_{x+\hat{1}} .
$$

Then for the partition function one writes

$$
\begin{aligned}
Z & =\operatorname{Tr} \exp (-\beta H) \\
& =\lim _{N \rightarrow \infty} \operatorname{Tr}\left[\exp \left(-\varepsilon \beta H_{1}\right) \exp \left(-\varepsilon \beta H_{2}\right)\right]^{N}
\end{aligned}
$$

where $\beta=1 / T$ is the inverse temperature and $\varepsilon \beta=\beta / N$ is the lattice spacing in Euclidean time. Inserting complete sets of eigenstates $|1\rangle$ and $|-1\rangle$ of $\sigma_{x}^{3}$ between the factors $\exp \left(-\varepsilon \beta H_{i}\right)$ one converts the partition function into a $(d+1)$-dimensional path integral of Ising-like variables

$$
Z=\prod_{x, t} \sum_{s(x, t)= \pm 1} \exp (-S)
$$

The action is a sum of plaquette couplings

$$
\begin{aligned}
& S=\sum_{x=2 m, t=2 p} S[s(x, t), s(x+\hat{1}), s(x, t+1), s(x+\hat{1}, t+1)] \\
& +\sum_{x=2 m+1, t=2 p+1} S[s(x, t), s(x+\hat{1}), s(x, t+1), s(x+\hat{1}, t+1)],
\end{aligned}
$$

and the plaquette Boltzmann factors are given by a $4 \times 4$ transfer matrix

$$
\exp \left(-S\left[s_{1}, s_{2}, s_{3}, s_{4}\right]\right)=\left\langle s_{1} s_{2}\left|\exp \left(-\varepsilon \beta J \vec{S}_{x} \cdot \vec{S}_{x+\hat{\mu}}\right)\right| s_{3} s_{4}\right\rangle
$$

For the spin-1/2 Heisenberg Hamiltonian, only six of the sixteen elements of the transfer matrix are non-zero, namely those that leave the 3-component of spin unchanged. 
The 2D Heisenberg model is of interest because it describes the undoped precursor insulators of high temperature superconductors, and it has been studied with various Monte Carlo techniques [3], including a very efficient loop cluster algorithm [4,5]. The cluster algorithm has led to a very precise determination of the low energy parameters of the Heisenberg model [5], consistent with the chiral perturbation theory treatment of Hasenfratz and Niedermayer [6]. The loop cluster algorithm has a well-defined continuous-time limit, as discussed below. The computational advantage of operating directly in the time continuum allows us to verify the predictions of chiral perturbation theory at temperatures that are inaccessible with the conventional discrete-time algorithm. The results for the low energy parameters of the theory are consistent with previous calculations [5] as well as with experimental data on precursor insulators of high temperature superconductors [7, 8].

The loop cluster algorithm in discrete time is implemented on a space-time lattice with $(L / a)^{2}$ spatial points and $4 N$ time slices, with a $\pm 1 / 2$ spin state defined at each lattice point. Both in space and in Euclidean time periodic boundary conditions are used. Decomposing the Hamiltonian with the Trotter formula allows us to define an action for each lattice configuration. Loops are built up according to a set of rules dictating the loop "flow" through each plaquette. After a loop is built, every spin on the loop is flipped. The loop-building rules are calibrated to satisfy the condition of detailed balance, ensuring that the path configuration space is sampled in proportion to the statistical weight of each configuration.

The discrete-time rules, together with their continuum analogs, are summarized in Table घ. Time is in the vertical direction for the plaquettes displayed here. The essence of the loop cluster algorithm is that we assign a "flow" to each legal plaquette, that is, to each plaquette with a non-zero entry in the transfer matrix and hence with finite action. In the case of the "optional decay" plaquettes the flow can proceed in either the space or the time direction; we choose the probability for proceeding in the space direction so that the entire algorithm satisfies detailed balance. Starting at a random site in space-time, we connect flows until the loop closes, and then all spins on the loop are flipped. The cluster-building rules ensure that the cluster paths always close. 
The continuum limit is determined by first observing that jumps from one spin site to a neighbor (the "optional" plaquettes in the table) are sporadic and of measure zero,

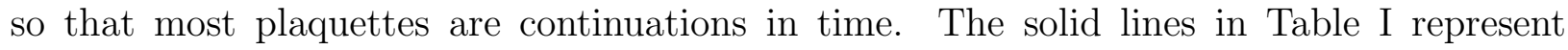
continuously spin up states, and the dotted lines are continuously spin down states. The forced transition plaquettes become "bonds" in the continuum limit, and the flow is required to travel along the bond and reverse direction in the continuum paradigm. The optional decay plaquettes become Poisson-like decays between unlike spins; the probability of a jump in the discrete case becomes a probability per unit time in the continuum case. The duration of a path segment on a given spin site is thus distributed like the lifetime of a radioactive nucleus: the more decay channels available, the more likely the path will terminate in a given amount of time. The essential difference between the discrete-time and continuoustime implementation is that the former requires us to store spin state information for each of the points of the space-time lattice, while the latter requires us only to store the transition times for each spin site (plus an extra bit to record the state at $t=0$ ). In the code developed here, all the transition times were maintained in a large array. A cyclic double-linked-list storage technique was implemented to ensure that the overhead associated with inserting and deleting time values was minimized.

A significant advantage of the loop cluster algorithm is that it allows for the implementation of improved estimators. We found that the improved estimators for susceptibility, staggered susceptibility, and internal energy density all have easily determined continuum limits.

The continuous-time algorithm with improved estimators was implemented in a FORTRAN code [9]. Instead of the node-by-node method of the discrete-time algorithm, the continuous-time algorithm is based on the exponential distribution of decay times. Starting at a randomly selected space-time location in the lattice, the neighborhood of the current endpoint of the path is surveyed. The first job of the survey is to determine the "horizon", which is the time of the next forced transition. The other purpose of the survey is to determine the number and duration of decay channels - i.e. sites with opposite spin 
unvisited by the current path. The time of the nearest change in the neighborhood is called the "waypoint." A pseudo-random number is generated, and a decay-point is computed according to the exponential distribution corresponding to the number of decay channels. The current time marker is moved to the closer of the waypoint or the decay-point. If the marker reaches the waypoint, then the waypoint and decay-point determination is repeated. If the marker reaches either the horizon or a decay-point, then the path moves to the appropriate neighbor, selected randomly from several channels if necessary. The process continues until the path closes.

In general terms, the computer time required to run a simulation for a given volume and temperature is roughly comparable for the discrete code (at a usable time-granularity) and the continuum code, but the continuum code requires substantially less storage space. The real advantage of the continuum code is that it obviates the need to conduct the several runs of successively finer time-granularity needed for extrapolation to the continuum limit, thus obviating a costly dimension in the simulation procedure.

In the ground state of the 2D Heisenberg antiferromagnet the staggered magnetization develops an expectation value, and hence the $O(3)$ spin rotational symmetry gets spontaneously broken down to $O(2)$. The low energy excitations of the systems are spinwaves (so-called magnons) which are the Goldstone bosons of the spontaneously broken $O(3)$ symmetry. Using symmetry arguments, chiral perturbation theory makes very strong predictions for magnon dynamics at low temperatures, with three low energy parameters as unknown constants. These parameters are the staggered magnetization $\mathcal{M}_{s}$, the spin wave velocity $\hbar c$, and the spin stiffness $\rho_{s}$. The predictions of chiral perturbation theory at extremely low temperatures were checked using a range of square volumes with side length $L / a=6,8 \ldots 20$ and a range of inverse temperatures $\beta J=1,2,5,10,20,30,40,50,80,100$. Note that the very small temperatures $T \approx 0.01 J$ are inaccessible to the discrete-time code, largely due to storage limitations. In every case 100,000 configurations were generated after a thermalization period of 1000 configurations.

Several of the key predictions of chiral perturbation theory were directly verified with 
this code. In particular, the energy spectrum is that of an $O(3)$ rotor, with energy levels characterized by an integer spin $j$, having degeneracy $2 j+1$ and energy levels distributed as $j(j+1)$. Finite volume effects are computed as expansions in $\hbar c / \rho_{s} L$, in units where the lattice spacing $a=1$. The energy spectrum is computed in reference [6] to be

$$
\begin{aligned}
& E_{j}-E_{0}= \\
& j(j+1) \frac{(\hbar c)^{2}}{2 \rho_{s} L^{2}}\left[1-\frac{\hbar c}{\rho_{s} L} \frac{3.900265}{4 \pi}+O\left(L^{-2}\right)\right] .
\end{aligned}
$$

Including only the leading term in the partition function, the uniform susceptibility approaches the form

$$
\chi_{u} \underset{T \rightarrow 0}{\longrightarrow} \frac{6}{L^{2} T} \exp \left(-\frac{(\hbar c)^{2}}{\rho_{s} L^{2} T}\right),
$$

and the staggered susceptibility goes to the temperature-independent form

$$
\chi_{s} \underset{T \rightarrow 0}{\longrightarrow} \frac{2 \mathcal{M}_{s}^{2} \rho_{s}}{(\hbar c)^{2}} L^{4}\left[1+3 \frac{\hbar c}{\rho_{s} L} \frac{3.900265}{4 \pi}+O\left(L^{-2}\right)\right] .
$$

These functional forms in the large $\beta$ limit, including both volume and temperature dependence, were verified for uniform and staggered susceptibility. In addition, the staggered susceptibility is shown to plateau at large $\beta$ as predicted by chiral perturbation theory.

An independent fit for the chiral perturbation theory parameters $\mathcal{M}_{s}, \hbar c$, and $\rho_{s}$ gives excellent agreement with the results of [5], as shown in Table [1]. This fit required that the partition function of the $O(3)$ rotor be included in its entirety, instead of including just the leading term, as the limiting forms in equations (8) and (9) employ. In addition, only inverse temperatures $\beta J \geq 10$ were used in the fit, since the rotor approximation is valid only for very small temperatures. In order to reproduce the accuracy of the fit in reference [5], it was necessary to find fitted values for the coefficients of the quadratic terms in the expressions for energy and staggered susceptibility, that is, the coefficients of the terms $\left(\hbar c / \rho_{s} L\right)^{2}$ in the expressions above. Note that the agreement between the current work and reference [5] is particularly remarkable because they are derived for different volume-temperature regimes. Reference [5] was concerned with the "cubical" regime $T L / \hbar c \cong 1$, while the 
current study focuses on the "cylindrical" regime $T L / \hbar c<<1$. Quadratic coefficients for

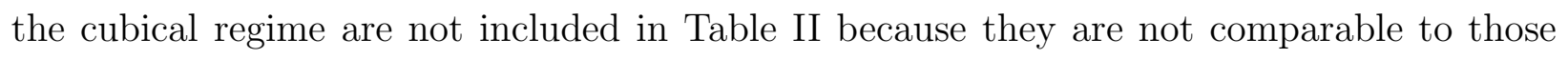
in the cylindrical regime. The five parameter values in Table $\mathbb{M}$ resulted in a goodness-of-fit $\chi^{2} /$ d.o.f. $=1.5$. Figures 1(a) and 1(b) show the fit for uniform and staggered susceptibility, respectively. Solid lines represent the fitted chiral perturbation theory result. Circles and error bars are displayed at each simulation point. The fit is quite good for $\beta J \geq 10$.

In conclusion, we find that the continuous-time formulation discussed here provides a superior method for evaluating path integrals that arise in the study of discrete quantum systems. Using this method allows us to validate the chiral perturbation theory results for the Heisenberg antiferromagnet in the extreme low temperature regime. In discrete Euclidean time a loop cluster algorithm has also been constructed for lattice fermions [10]. Our continuous time method can be applied to this problem as well. In fact, in principle it works for any discrete quantum system, even if a cluster algorithm may not be available. For example, for a nonrelativistic particle living in a finite volume one can formulate the path integral using the discrete momentum states. Similarly, for a lattice gauge theory with a compact gauge group one can work in the discrete basis of representations. In both cases this yields a well-defined path integral directly in the Euclidean time continuum.

We would like to thank P. Grassberger for asking a question that led to this project. We also thank E. Farhi and S. Gutmann for helpful discussions. This material is based upon work supported under a National Science Foundation Graduate Fellowship (BBB) and an Alfred P. Sloan Fellowship (UJW). This work has also been supported in part by funds provided by the U.S. Department of Energy (D.O.E.) under cooperative research agreement DE-FC02-94ER40818. 


\section{REFERENCES}

[1] E. Farhi and S. Gutmann, Ann. Phys. 213 (1992) 182.

[2] M. Suzuki, Prog. Theor. Phys. 56 (1976) 1454.

[3] M.S. Makivić and H.-Q. Ding, Phys. Rev. B43 (1991) 3562.

[4] H.G. Evertz, G. Lana, and M. Marcu, Phys. Rev. Lett. 70 (1993) 875.

[5] U.-J. Wiese and H.-P. Ying, Z. Phys. B93 (1994) 147.

[6] P. Hasenfratz and F. Niedermayer, Z. Phys. B92 (1993) 91.

[7] G. Shirane et al., Phys. Rev. Lett. 59 (1987) 1613.

[8] M. Greven et al., Z. Phys. B96 (1995) 465.

[9] This code will be made available via the World-Wide Web. Check http://ctpa02.mit.edu/ bbbeard/ for details.

[10] U.-J. Wiese, Phys. Lett. B311 (1993) 235. 


\section{FIGURES}

FIG. 1. (a) Uniform susceptibility and (b) staggered susceptibility versus inverse temperature for various volumes. Solid lines are predictions from chiral perturbation theory with fitted parameters; circles with error bars are lattice simulations with continuous time. The fit is very good for $\beta J \geq 10$. 


\section{TABLES}

TABLE I. Summary of Plaquette Flow Rules. $+1 / 2$ and solid lines indicate spin-up sites; $-1 / 2$ and dotted lines are spin-down. The time direction is vertical. Flow rules for inverse plaquettes are analogous.

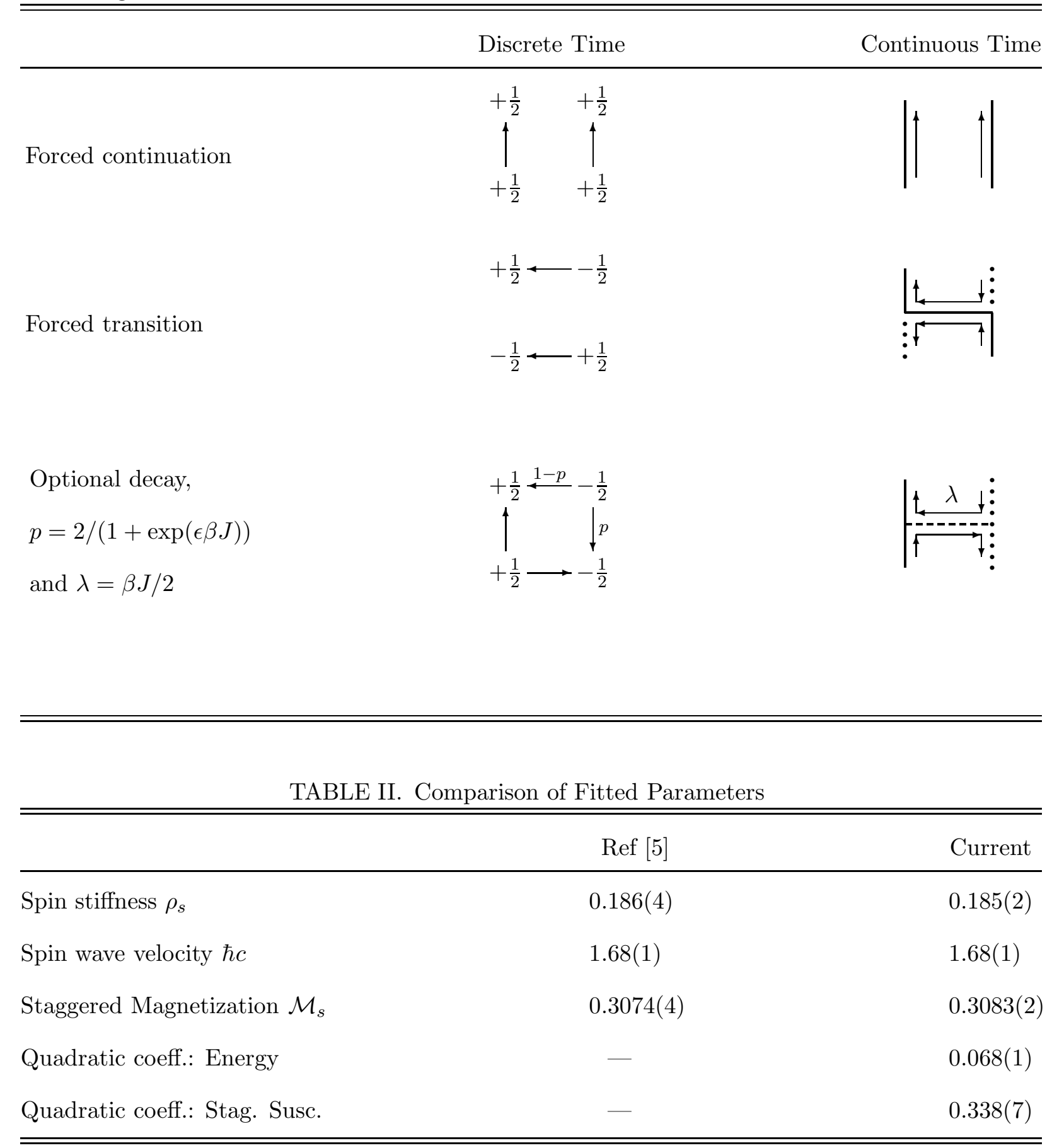




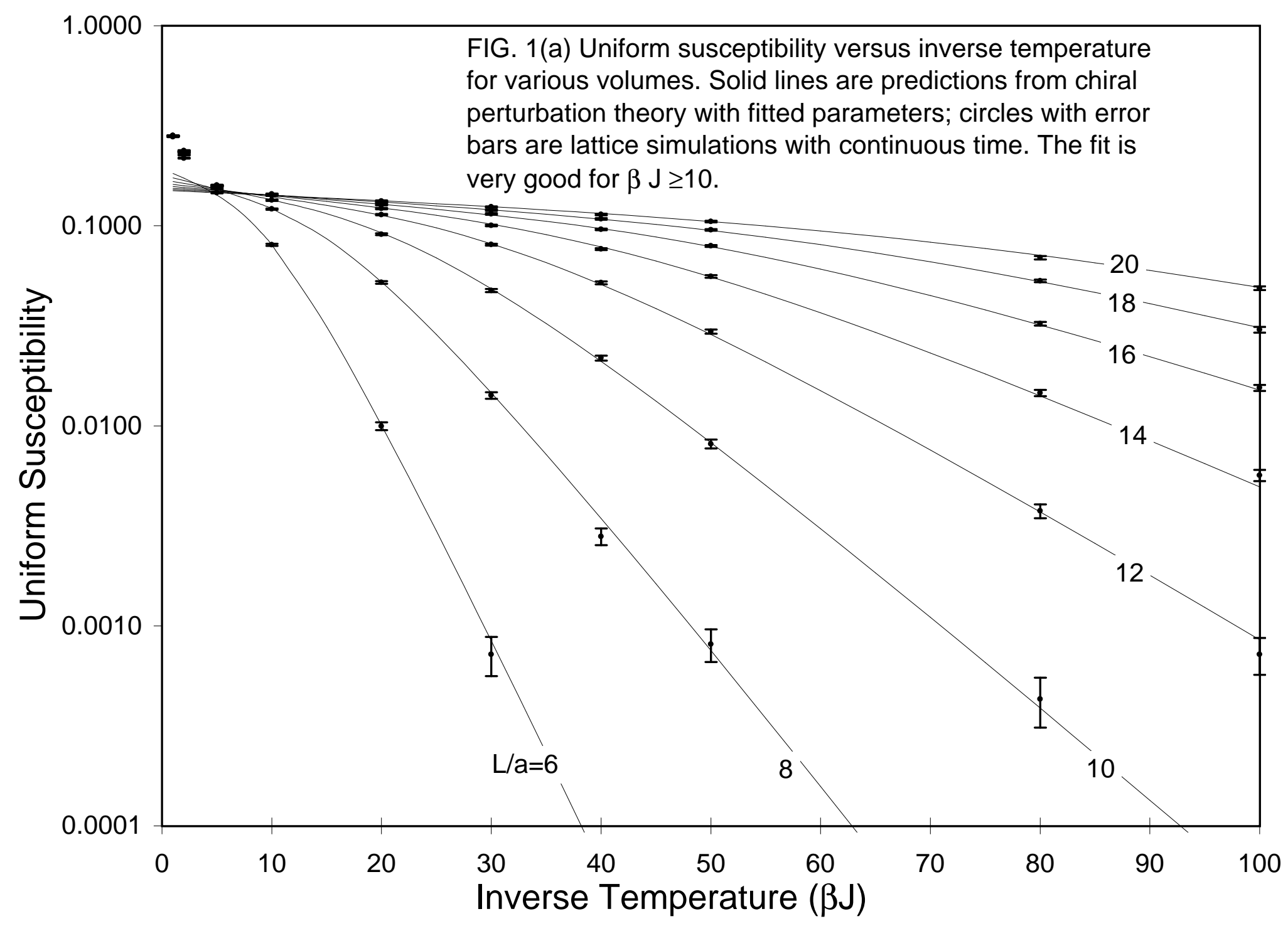




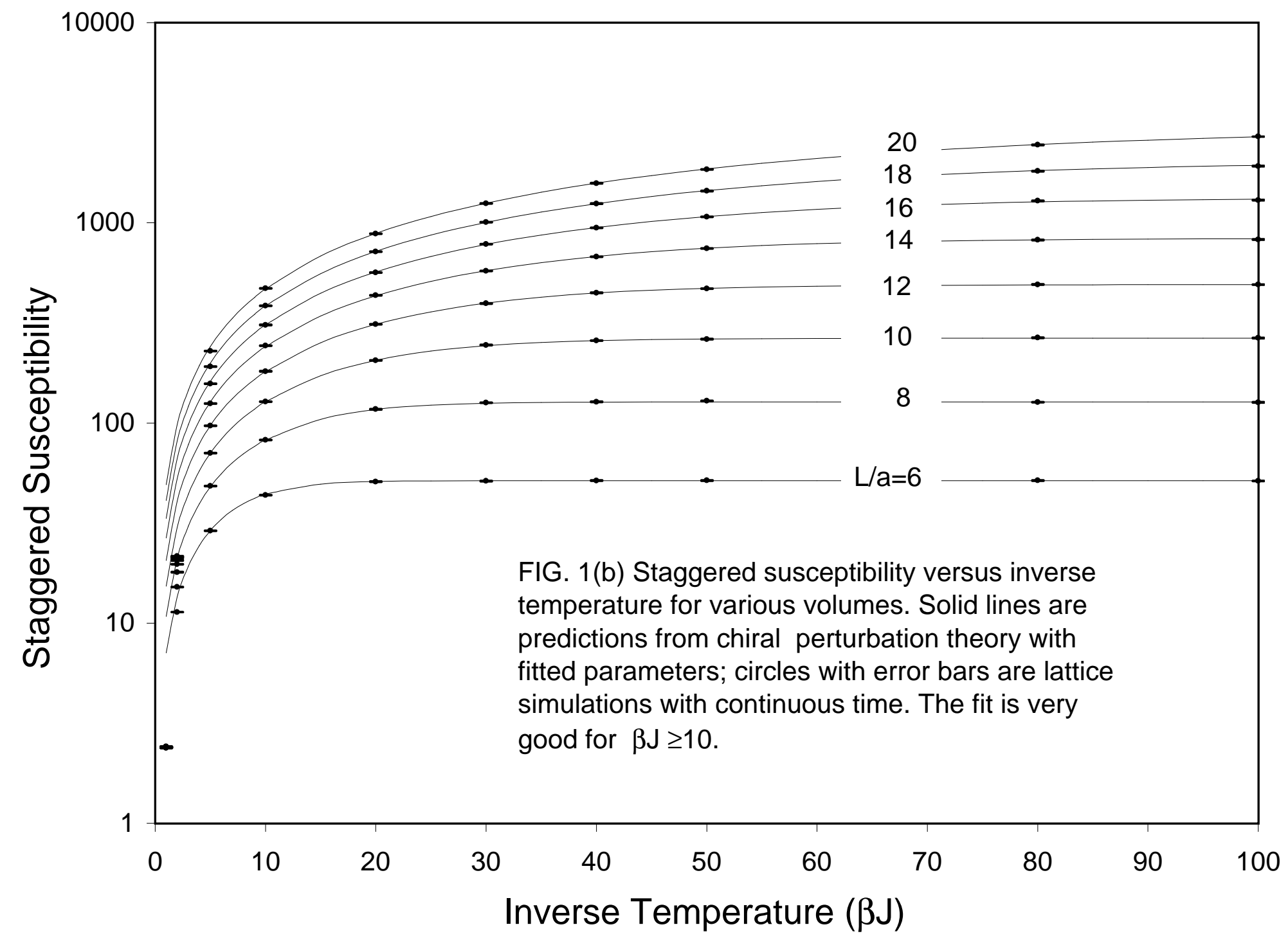

